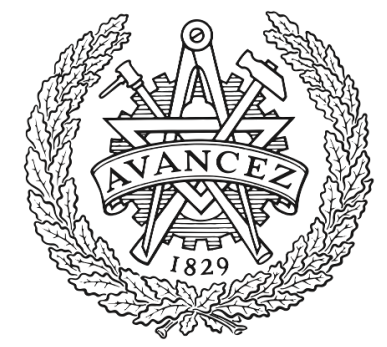

CHALMERS

UNIVERSITY OF TECHNOLOGY

\title{
Reconfigurable Transmitarray with Near Field Coupling to Gap Waveguide Array Antenna for Efficient 2D Beam Steering
}

Downloaded from: https://research.chalmers.se, 2023-04-26 10:41 UTC

Citation for the original published paper (version of record):

Vilenskiy, A., Makurin, M., Lee, C. et al (2020). Reconfigurable Transmitarray with Near Field Coupling to Gap Waveguide Array Antenna for Efficient 2D Beam Steering. IEEE Transactions on Antennas and Propagation, 68(12): 7854-7865. http://dx.doi.org/10.1109/TAP.2020.2998904

N.B. When citing this work, cite the original published paper.

(O2020 IEEE. Personal use of this material is permitted.

However, permission to reprint/republish this material for advertising or promotional purposes 


\title{
Reconfigurable Transmitarray with Near-Field Coupling to Gap Waveguide Array Antenna for Efficient 2D Beam Steering
}

\author{
Artem R. Vilenskiy, Member, IEEE, Mikhail N. Makurin, Chongmin Lee, Member, IEEE, and \\ Marianna V. Ivashina, Senior Member, IEEE
}

\begin{abstract}
A novel array antenna architecture is proposed that can enable 2D (full-space) radiation pattern control and efficient beam steering. This solution is based on a fixed-beam gap waveguide (GWG) array antenna and a reconfigurable transmitarray (TA) that are coupled in the radiative near field. An equivalent two-port network model of the coupling mechanism is presented and validated numerically. The desired $T A$ reconfiguration capability is realized by an $8 \times 8$ array of cavity-backed patch resonator elements, where two AlGaAs PIN-diodes are integrated inside each element providing a 1-bit phase shift. The TA is implemented in an 8-layer $\mathrm{PCB}$, which includes radiating elements, fixed phase-shifting inner-stripline sections, impedance matching and biasing circuitry. The combined antenna design is low-profile ( 1.7 wavelength) owing to the small separation between two arrays $(\sim 0.5$ wavelength), as opposed to conventional TAs illuminated by a focal source. The design procedure of the proposed architecture is outlined, and the measured and simulated results are shown to be in good agreement. These results demonstrate 23.5 - 25.2 GHz $-10-d B$ impedance bandwidth and $23.3-$ 25.3 GHz 3-dB gain bandwidth, a beam-steering range of $\pm 30^{\circ}$ and $\pm 40^{\circ}$ in the $E$ - and the $H$-plane with the gain peak of $17.5 \mathrm{dBi}$, scan loss $\leq 3.5 \mathrm{~dB}$ and $\mathrm{TA}$ unit cell insertion loss $\leq$ $1.8 \mathrm{~dB}$.
\end{abstract}

Index Terms - Phased antenna array, transmitarray, array lens, discrete lens, 1-bit phase-shifting, gap waveguide.

\section{INTRODUCTION}

$\mathrm{T}$ HE continued growth in data traffic in different commercial applications, including terrestrial wireless communi-

Manuscript received XX.XX.XX; Revised XX.XX.XX; accepted XX.XX.XX. Date of publication XX.XX.XX; date of current version XX.XX.XX. This research was carried out as an in-kind contribution to the ChaseOn Centre in Integrated Antenna Array project financed by Vinnova, Chalmers University of Technology, Royal Institute of Technology (Stockholm), Ericsson, Saab, Ruag Space, Keysight, and Gapwaves.

A. R. Vilenskiy is with the Electrical Engineering Department, Chalmers University of Technology, Gothenburg 41258, Sweden. He is also with RF Sensor Part of Samsung Research Institute Russia, Moscow, Dvintsev, 12, 127018, Russia, corresponding author (e-mail: artem.vilenskiy@chalmers.se).

M. N. Makurin is with RF Sensor Part of Samsung Research Institute Russia, Moscow, Dvintsev, 12, 127018, Russia. (e-mail: m.makurin@samsung.com).

C. Lee is with Smart Device Team of Samsung Research, Seoul, Seongchon-gil, 34, 06765, Republic of Korea. (e-mail: cm3109.lee@samsung.com).

M. V. Ivashina is with the Electrical Engineering Department, Chalmers University of Technology, Gothenburg 41258, Sweden (e-mail: marianna.ivashina@chalmers.se). cations [1], satellite communications [2], and automotive radars [3], requires mm-wave frequency antenna technologies with advanced beamforming capabilities. However, the design of such antenna systems has been proven challenging, due to the necessary trade-offs between demanding performance characteristics as well as the size and cost constraints [4]. One particular challenge is power losses in the antenna radiating structure and feed network, a problem that can severely degrade the performance at mm-wave frequencies. Hence, design decisions heavily rely on suitable fabrication technology and the type of the feed network.

Typically, mm-wave phased antenna arrays (PAAs) employ either corporate feed networks or spatial excitation (including transmitarrays or array lenses) [5] - [7]. The latter are usually characterized by lower losses, especially if the number of antenna array elements and frequency increase. However, they are bulky due to the focal source placed at the electrically large distance from the radiating aperture. On the contrary, corporate feed array antennas can be made low-profile using, e.g., multi-layer printed circuit board (PCB) [8] or low temperature co-fried ceramic technology [9]. Unfortunately, at $\mathrm{mm}$-wave frequencies the limitations due to high losses in a power distribution system become critical. To some extent, this problem can be minimized by employing an active architecture [10], though, making a design expensive and, in many cases, unsuitable for commercial products.

More recently, a gap waveguide (GWG) technology has been introduced for mm-wave applications. The GWG-fed array antennas can be realized with $\mathrm{H}$-plane waveguide splitblocks in several metal layers and have both low-loss and lowprofile characteristics [11] - [17]. To this day, due to the complexity of radiating element excitation control inside the GWG structures, such designs have been used mainly for fixed-beam applications, except for some recent works presenting leaky-wave antennas with frequency-dependent beam steering [18] - [20] and 1D electronic beam steering antennas [7], [21], [22].

An alternative low-profile beam-steering PAA was recently proposed in [23], where the varactor-tunable C-band transmitarray (TA) was fed by an electromagnetically coupled leaky-wave rectangular waveguide (WG) feed. Owing to the direct coupling approach, the demonstrated reduction in the overall thickness of the antenna was 10 -fold, as compared to 
TABLE I

COMPARISON OF DIFFERENT PAA ARCHITECTURES EMPLOYING TAS

\begin{tabular}{|c|c|c|c|c|c|c|c|c|c|c|c|c|c|c|}
\hline \multirow{2}{*}{$\begin{array}{l}\text { TA } \\
\text { excitation } \\
\text { method }\end{array}$} & \multirow[t]{2}{*}{$\begin{array}{l}\text { Feed } \\
\text { type }\end{array}$} & \multirow{2}{*}{$\begin{array}{l}\text { Beam } \\
\text { steering } \\
\text { implemen- } \\
\text { tation }\end{array}$} & \multirow{2}{*}{$\begin{array}{l}\text { Full } \\
\text { system } \\
\text { design }\end{array}$} & \multirow{2}{*}{$\begin{array}{l}\text { Scalabil- } \\
\text { ity in } \\
\text { array size }\end{array}$} & \multirow{2}{*}{$\begin{array}{l}\text { Power } \\
\text { handling } \\
\text { per UC, } \\
\text { mW }\end{array}$} & \multirow[t]{2}{*}{$\begin{array}{l}\mathrm{f}_{0}, \\
\mathrm{GHz}\end{array}$} & \multirow{2}{*}{$\begin{array}{l}\text { Polaization / } \\
\text { switcha- } \\
\text { ble }\end{array}$} & \multirow{2}{*}{$\begin{array}{c}-10-\mathrm{dB} \\
\text { imp. } \\
\text { band- } \\
\text { width, } \\
\%\end{array}$} & \multirow{2}{*}{$\begin{array}{l}\text { 3-dB } \\
\text { gain } \\
\text { band- } \\
\text { width, } \\
\%\end{array}$} & \multicolumn{3}{|c|}{ Efficiency loss factors, dB } & \multirow{2}{*}{$\begin{array}{l}\text { Peak total } \\
\text { antenna } \\
\text { efficiency, } \\
\%\end{array}$} & \multirow{2}{*}{$\begin{array}{l}\text { Longi- } \\
\text { tudina } \\
\text { size, } \lambda\end{array}$} \\
\hline & & & & & & & & & & $\begin{array}{l}\text { UC ins. } \\
\text { loss }\end{array}$ & \begin{tabular}{|l|} 
Spillover/ \\
end \\
leakage \\
\end{tabular} & $\begin{array}{l}\text { Phase } \\
\text { and } \\
\text { taper }\end{array}$ & & \\
\hline $\begin{array}{l}\text { by a focal } \\
\text { source } \\
{[34]-} \\
{[36],[39]}\end{array}$ & $\begin{array}{c}\text { Horn }(\mathbf{H}) \\
{[35],[39]} \\
\text { Focal array } \\
\text { (A) [39] }\end{array}$ & $\begin{array}{c}\text { 1-bit phase } \\
\text { control } \\
\text { (AlGaAs } \\
\text { PIN diodes) }\end{array}$ & $\begin{array}{l}\text { Separately } \\
\text { for TA } \\
\text { and feed }\end{array}$ & Direct & $\leq 50$ & $\begin{array}{l}9.8 \\
{[35]}\end{array}$ & $\begin{array}{c}\text { Linear / } \\
\text { No }\end{array}$ & $\begin{array}{l}13.1 \\
\text { (UC) }\end{array}$ & 15.8 & 2.1 & $1-$ & 6.2 & $\begin{array}{c}15 \\
@ 9.8 \mathrm{GHz}\end{array}$ & 8.1 \\
\hline $\begin{array}{l}\text { by an } \\
\text { integrated } \\
\text { feed [23] }\end{array}$ & $\begin{array}{c}\text { Leaky- } \\
\text { wave } \\
\text { WG } \\
\text { array }\end{array}$ & $\begin{array}{c}\text { Continuous } \\
\text { phase control } \\
\text { (AFA with } \\
\text { GaAs varactors) }\end{array}$ & $\begin{array}{l}\text { TA-feed } \\
\text { co-design }\end{array}$ & $\begin{array}{l}\text { Feed 1e- } \\
\text { design }\end{array}$ & $\leq 5$ & 4.85 & $\begin{array}{c}\text { Linear / } \\
\text { No }\end{array}$ & \begin{tabular}{|c|} 
Not \\
available
\end{tabular} & 9.3 & 3.5 & 1.1 & 0.25 & @ $\begin{array}{c}31 \\
4.8 \mathrm{GHz}\end{array}$ & 0.75 \\
\hline $\begin{array}{l}\text { using } \\
\text { NFC } \\
\text { (this } \\
\text { work) } \\
\end{array}$ & $\begin{array}{l}\text { GWG } \\
\text { array }\end{array}$ & $\begin{array}{c}\text { 1-bit phase } \\
\text { control } \\
\text { (AlGaAs } \\
\text { PIN diodes) }\end{array}$ & $\begin{array}{l}\text { Separately } \\
\text { for TA } \\
\text { and feed }\end{array}$ & $\begin{array}{l}\text { Feed } \\
\text { scaling }\end{array}$ & $\leq 50$ & 24.5 & $\begin{array}{c}\text { Linear / } \\
\text { No }\end{array}$ & 7 & 8.2 & 1.8 & $\begin{array}{c}\text { Not } \\
\text { applicable }\end{array}$ & 4 & $\begin{array}{c}20 \\
\text { @ } 25 \mathrm{GHz}\end{array}$ & 1.7 \\
\hline
\end{tabular}

the conventional TA with a focal source (see Table I). However, the insertion loss of the varactor-loaded unit cell (UC) was high, i.e. $3.5 \mathrm{~dB}$, even for relatively low frequencies. Other design drawbacks are limited transmitted power, a complicated 3D structure of the UC requiring laborious assembling, and the time-consuming design procedure. These factors significantly limit the overall antenna efficiency and scalability to larger-scale arrays and higher frequencies.

In this work, we propose a solution to the above-mentioned problems of conventional TAs with a focal source (i.e. large size/volume) and the TA with integrated WG feed (i.e. high insertion loss, and poor scalability). The proposed new architecture of a mm-wave beam-steering PAA is based on the combination of a high-efficiency fixed-beam GWG array and a controllable TA with integrated PIN diodes. These arrays are mutually coupled in the radiative near-field region (Fig. 1), with the $\sim 0.5 \lambda$ distance between them (where $\lambda$ is the freespace wavelength). In this near-field coupling (NFC) architecture, the required joint arrays operation is guaranteed by impedance-matching each array to free space for the broadside radiation regime. Thus, the TA can be considered as a supplementary beam-steering structure for the fixed-beam GWG array that allows for 2D (full-space) radiation pattern control. At the same time, the GWG array can be seen as a low-loss integrated feed of the TA that enhances its efficiency and scalability. This design approach has similarities with the approach of the transmission and reflection grid amplifier [24], where the active grid represents a supplementary (spatial signal amplification) structure in waveguide. In this study, we focus on the beam-steering performance of PAA systems and the associated implementation challenges. It should be mentioned that the NFC concept was recently applied in [25] to the case of a planar phase-shifting surface excitation, though without a quantitative description.

This paper is organized as follows. In Section II, we explain the operation principle of the TA and give the equivalent twoport network representation. The beam-steering implementation concept, as realized in the example $8 \times 8$ antenna design, is also discussed here. Section III describes the design flow of the total $8 \times 8$ PAA system and presents numerical and experimental results covering the analysis of the GWG array and the TA UC, as well as their NFC operation. In Section IV, the overall antenna performance is studied experimentally. Finally, Section $\mathrm{V}$ formulates main conclusions.

\section{ANTENNA ARRAY ARCHITECTURE EMPLOYING NFC}

\section{A. Operation Principle}

A block diagram of the proposed PAA architecture is presented in Fig. 1. The fixed-beam array comprises $N=N_{x} N_{y}$ elements with the inter-element spacings $d_{x}, d_{y}$, where $N_{x}, N_{y}$ are the numbers of elements along $x$ - and $y$-axis, respectively. The controllable TA, in turn, has the same number and arrangement of elements. Both arrays are coupled in the radiative near-field region (the Fresnel region). The controllable TA comprises three main parts: (i) the array of RX antenna elements which are coupled to the fixed-beam array; (ii) the array of reconfigurable TX antenna elements radiating into free space; and (iii) the inner structure interconnecting RX and TX elements inside each UC, which also integrates the required fixed phase shifting elements and DC circuitry. The distance between the fixed-beam array aperture and the RX aperture of the TA is defined by $D_{\text {coup }}$.

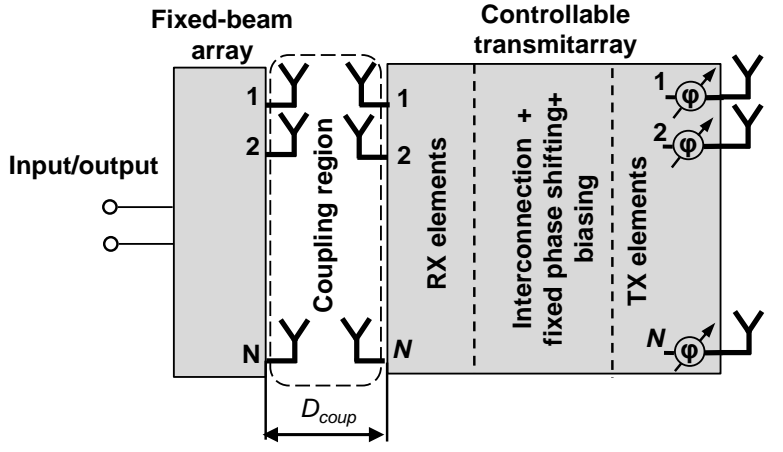

Fig. 1. A block diagram of the proposed PAA architecture. 
Electromagnetic coupling between two arrays exists owing to the fact that an infinite equiphase periodic structure with $d_{x}<\lambda$ and $d_{y}<\lambda$ excites a pair of the dominant Floquet modes [26], which are the propagating plane waves. At the same time, the higher-order Floquet modes do not propagate, while giving rise to the reactive field distribution above the periodic structure. Since the higher-order modes typically attenuate very fast with the distance above the periodic structure, the radiated field is almost a pure plane wave at already about $0.5 \lambda$. Although the above assumption is strictly valid for infinite arrays, it has shown to provide sufficient accuracy for the representation of the radiative near field of electrically large finite arrays [27]. When the distance above the finite array aperture increases, we approach the lower boundary of the Fraunhofer region [28], and the radiated field diverges transforming into a spherical wave. Thus, the following inequality for $D_{\text {coup }}$ can provide us the TA plane-wave excitation by the fixed-beam array near field:

$$
0.5 \lambda \leq D_{\text {coup }} \ll 2 L_{P A A}^{2} / \lambda,
$$

where $L_{P A A}$ is the maximum linear size of the radiating structures. If (1) is satisfied and near fields of the arrays are polarization matched, then both vector near-field structures are fully matched. In this case, the coupling mechanism can be analyzed using the equivalent two-port network in Fig. 2, where the cascaded two-port networks represent the corresponding parts of the coupled system. This model assumes periodic boundary conditions (PBC) imposed on the system sidewalls, where all parts are interacting through a single dominant Floquet mode (linear polarization) only. Then, according to [26], the following relations are valid for the coupled system $S$ matrix entries:

$$
\begin{gathered}
S_{11}^{N F C}=S_{11}^{F B}+\frac{S_{21}^{F B} S_{12}^{F B} S_{11}^{R X} \exp \left(-j 2 k_{0} D_{\text {coup }}\right)}{1-S_{22}^{F B} S_{11}^{R X} \exp \left(-j 2 k_{0} D_{\text {coup }}\right)}, \\
S_{21}^{N F C}=\frac{S_{21}^{F B} S_{21}^{R X} \exp \left(-j k_{0} D_{\text {coup }}\right)}{1-S_{22}^{F B} S_{11}^{R X} \exp \left(-j 2 k_{0} D_{\text {coup }}\right)},
\end{gathered}
$$

where $k_{0}$ is the free-space wavenumber. The numbering of ports for each two-port network starts from the left side. In (2), (3), the exponential terms describe the Floquet mode broadside propagation inside the coupling region. A verification of the proposed representation will be discussed in Section III.C.

From (2) we can see that the sufficient condition for the coupled system impedance matching is the matching of the

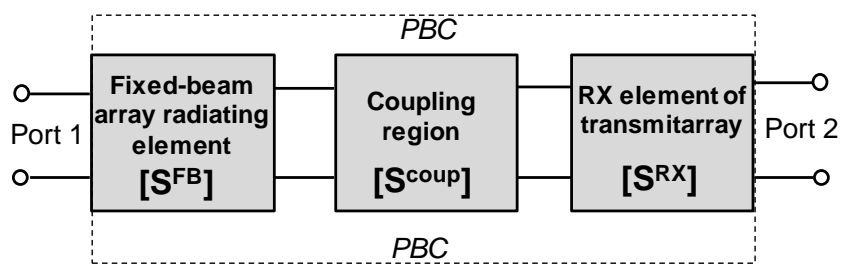

Fig. 2. The equivalent two-port network representation of the near-field coupled UCs, where the cascaded two-port networks interact only through a single dominant Floquet mode. fixed-beam and the TA RX elements for the broadside radiation regime in free space (i.e. $S^{F B}{ }_{11}=S^{R X}{ }_{11}=0$ ). The discussed operation principle provides an important feature of the design flow - two arrays can be developed independently for the matched operation in free space. Of course, the impedance matching of the full antenna system will also depend on the matching of the TA TX aperture.

The main advantage of the proposed structure over the architecture with a focal source [29] — [40] is a significant longitudinal size reduction provided by (1). It should be noted that replacing a single focal source by a focal array [38], [39] allows for a more compact design, though at the cost of the dramatically reduced antenna efficiency (see Table 1).

\section{B. Beam Steering Implementation Concept: 1-Bit Phase Control and "Virtual Focus" Approach}

There are several implementation approaches for TA beam steering, where most advanced of them use an intermediate phase-shifting section realizing the antenna-filter-antenna (AFA) concept. In this way, one can employ array antenna elements with 2-bit phase control using PIN diode or MEMS switches (cf. [29], [30]) in order to provide efficient beam steering. Alternatively, varactor-loaded AFA cells as in [23], [31], [32] can be used to realize the full $360^{\circ}$ phase control. However, the common drawbacks of the AFA implementations are complicated designs, high cost, and laborious assembling. Another beam steering method is based on reconfigurable UCs, where radiating elements are integrated with active controllable devices such as varactor [33] or PIN diodes [34] — [40]. This approach results in low-profile TA designs with minimized UC insertion loss. The PIN diode-based designs with a 1-bit phase resolution are often preferred in commercial products (cf. the example of the PIN diode integrated Oslotted patch TAs in [34] - [36]), thanks to the lowest insertion loss, the simplicity of control circuitry, and high power handling capabilities.

The above reasons have motivated the beam steering concept as used in the present work. For its optimal implementation in our design, several modifications were required because of the plane-wave excitation of the TA that causes phase quantization mirror lobes of the PAA radiation patterns, due to the 1-bit phase resolution [41], [42]. To eliminate this problem, an additional fixed phase shift distribution should be generated over the TA TX aperture. The detailed description is given below.

Fig. 3 schematically demonstrates two excitation scenarios, when the TA is illuminated by a plane wave (red line) or by a spherical wave of a focal source (blue curves). To steer the beam to the $\left(\theta_{s}, \varphi_{s}\right)$ direction (where $\theta_{s}, \varphi_{s}$ are the beam steering elevation and azimuth angles, respectively), the following phase values $\varphi_{i j}^{0}$ should be compensated by the phase shifter of the $(i, j) \mathrm{UC}$ :

$$
\varphi_{i j}^{0}=\varphi_{i j}^{e}+\varphi_{i j}^{i n}+\varphi_{i j}^{s},
$$

where $\varphi_{i j}^{e}$ is an excitation phase shift; $\varphi^{i{ }_{i j}}$ is the inner (fixed) phase shift of the $(i, j) \mathrm{UC}$; and $\varphi_{i j}^{s}$ is the beam steering phase 


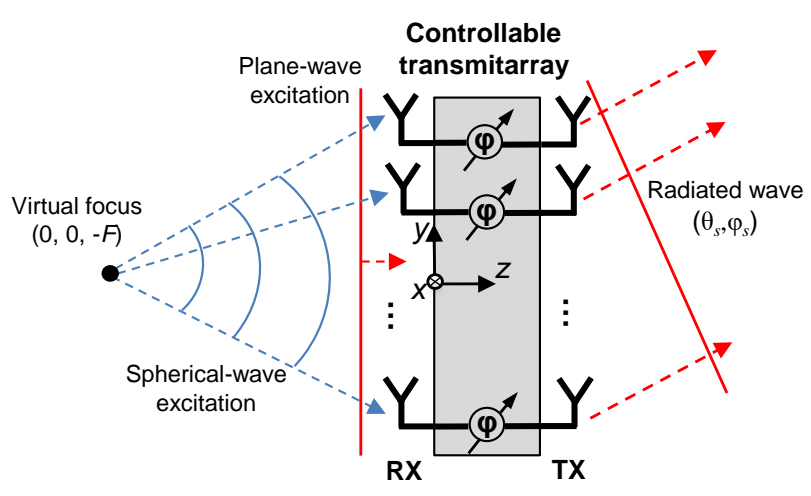

Fig. 3. TA excitation scenarios.

shift:

$$
\varphi_{i j}^{s}=\frac{2 \pi}{\lambda} \sin \left(\theta_{s}\right)\left(x_{i}^{T X} \cos \left(\varphi_{s}\right)+y_{j}^{T X} \sin \left(\varphi_{s}\right)\right) .
$$

In (3), $x^{T X}{ }_{i}, y^{T X}$ are the center coordinates of the $(i, j) \mathrm{TX}$ UC. The TA phase state $\varphi^{P S}{ }_{i j}$ for the case of the 1-bit phase control can be calculated as follows:

$$
\varphi_{i j}^{P S}=\left\{\begin{array}{l}
0,-\pi / 2 \leq \operatorname{Arg}\left(\exp \left(-j \varphi_{i j}^{0}\right)\right)<\pi / 2 \\
\pi, \text { otherwise }
\end{array} .\right.
$$

Finally, the resulting full phase $\varphi^{\Sigma_{i j}}$ of the $(i, j) \mathrm{UC}$ becomes

$$
\varphi_{i j}^{\Sigma}=\varphi_{i j}^{e}+\varphi_{i j}^{i n}+\varphi_{i j}^{P S} .
$$

In the case of the uniform inner phase distribution and the plane-wave excitation $\left(\varphi^{i n}{ }_{i j}=\varphi_{i j}^{e}=0\right)$ the term $\varphi^{s}$ ij is the only one which remains in the right-hand side of (4). This simplification, however, leads to the aforementioned parasitic effects during beam steering with the 1-bit phase quantization. Physically, these effects can be illustrated by considering the beam steering in one of the principal array planes. Then, according to (6) and (7), $\varphi \Sigma_{i j}=0$ or $\pi$, and thus the TX aperture has a real-valued excitation distribution. The normalized TX elements excitation amplitudes take the values -1 and 1 , with the period along the PAA of $\lambda / \sin \left(\theta_{s}\right)$. As the far-field pattern represents the Fourier transform of the source distribution [28], we will inevitably see the pattern with two symmetrically allocated identical lobes: the first lobe is the main lobe corresponding to the desired beam-steering direction $\theta_{s}$, whereas the second lobe is the parasitic mirror lobe at $-\theta_{s}$. In [42], the parasitic effects, corresponding to the 1-bit phase quantization, were thoroughly addressed analytically, and several compensation approaches, employing nonlinear inner phase distributions, were considered. In the presented study we adopt socalled "virtual focus" approach [42] which assumes that the TA is virtually excited by a focal source at $(0,0,-F)$ position (see Fig. 3):

$$
\varphi_{i j}^{i n}=-\frac{2 \pi}{\lambda}\left(\sqrt{x_{i}^{R X 2}+y_{j}^{R X 2}+F^{2}}-F\right) .
$$

In (8), $x^{R X}{ }_{i}, y^{R X}{ }_{j}$ are the center coordinates of the $(i, j) \mathrm{RX}$ $\mathrm{UC} ; F$ is the focal distance.

The required inner phase shift distribution can be realized, for example, by time delay lines of various lengths allocated inside the inner interconnecting structure of the TA (Fig. 1). The length increment $\Delta L_{i j}$ of $(i, j)$ UC's time delay line is computed from the relation:

$$
\Delta L_{i j}=\left(\max \left(\varphi_{i j}^{e}\right)-\varphi_{i j}^{e}\right) / \beta,
$$

where $\beta$ is the phase constant of the time delay line.

\section{Antenna system configuration and radiation model}

In this study, the following TA configuration will be considered: $N_{x}=N_{y}=8, d_{x}=d_{y}=0.6 \lambda_{0}=7.5 \mathrm{~mm}$ (chosen such to meet the grating lobe free condition up to $\pm 42^{\circ}$ from the broadside), where $\lambda_{0}$ is the operation wavelength corresponding to the central frequency $f_{0}=24 \mathrm{GHz}$.

The proposed architecture has the free parameter $F$, which determines the inner phase distribution of the TA according to (8). As shown in [42], $F$ has a major impact on the side lobe level (SLL) and directivity. The problem of the optimum inner phase distribution was studied in [42] using the TA radiation model and the spectral representation of array factor. The optimum $F$ was found applying the minimum SLL criterion that was $F_{\text {opt }} \approx 67 \mathrm{~mm}$ for the $8 \times 81$-bit TA. In this study, $F$ was reduced to $60 \mathrm{~mm}$ to achieve better average SLL. This correction should not be confusing, since in [42] the optimum relations were obtained using the stationary phase method and are strictly valid for $N_{x, y} d_{x, y} / \lambda \gg 1$. As a result, the expected maximum SLLs are below $-8 \mathrm{~dB}$ with the $3.5 \mathrm{~dB}$ maximum scan loss over the $2 \mathrm{D}$ scan range of $\pm 40^{\circ}$.

The TA radiation model using the aperture diffraction theory [28] was employed in this study, thus representing the TA by a radiating aperture with a piecewise constant field distribution. This model can be written as the multiplication of the element and space factors, where the former corresponds to the Huygens source according to the chosen relation between aperture electric and magnetic fields (see the details in [42]).

\section{NFC ANTENNA SySTEM DESIGN}

The design flow of the proposed PAA architecture comprises the following main steps: (i) development of a fixed-beam GWG array optimized for the broadside radiation in free space; (ii) design of a TA with an RX aperture optimized for the broadside illumination and a TX aperture optimized for beam steering within the desired scan range; (iii) direct NFC of the two arrays by optimizing the value of $D_{\text {coup }}$. Below, we provide the details of each step.

\section{A. Fixed-Beam GWG Array}

A fixed-beam GWG array is composed of three aluminum layers that represent an $8 \times 8$ array of slot antenna elements, a ridge gap waveguide (RGW) distribution layer, and an intermediate resonator layer between them. Fig. 4(a) shows the full 3D design, and Figs. 4(b) - (d) illustrate its main building blocks, where we denoted the critical design parameres. The 
gap between the ridge and the upper metal plate is $0.3 \mathrm{~mm}$, and the gap between the resonator pins and the slotted plate is $0.8 \mathrm{~mm}$. Thicknesses of the RGW, resonator and slot antenna array aperture are $2 \mathrm{~mm}, 2 \mathrm{~mm}$, and $1 \mathrm{~mm}$, respectively. To match the slotted aperture with the RGW distribution layer, we used two matching groups of elements, denoted M1, M2 in Fig. 4(b). The group M1 represents $6.4 \times 2 \times 1.3 \mathrm{~mm}^{3}$ tuning ledges performed on the resonator metal plate. Elements M2 are $8 \times 2 \times 1.25 \mathrm{~mm}^{3}$ ledges formed on the bottom side of the slotted plate. All antenna parts have been manufactured using a $1 \mathrm{~mm}$ milling tool.

The array design procedure is in general similar to the GWG configuration from [12]. Nevertheless, a specific attention was paid to the modal content of the slotted aperture, since the GWG array UC is essentially the $2 \times 2$ slot subarray fed by a single RGW line. In [12], the separation between slots in the H-plane varies (i.e. the array is quasiperiodic). As a result, the higher-order propagating Floquet modes of the periodic slotted structure are excited. To avoid this, we designed the GWG array with the strictly periodic slots allocation (Fig. 4(d)). Fig. 5(a) demonstrates the simulated $S$ parameters of the $2 \times 2$ subarray inside the infinite array environment (Fig. 5(b)). The magnitudes of the transmission coefficients to the higher-order Floquet modes are below $-25 \mathrm{~dB}$, which is an order of magnitude lower compared to [12].

An experimental prototype of the fixed-beam GWG array was manufactured and experimentally characterized in the anechoic chamber. Fig. 6 demonstrates measured frequency responses of the input port reflection coefficient $\left(S_{11}\right)$ and broadside realized gain. A photograph of the disassembled antenna is shown in the inset of Fig. 6(b). The agreement between the simulated and measured results is good with the most noticeable differences near $21.5 \mathrm{GHz}$, where the measured $S_{11}$ level is above $-10 \mathrm{~dB}$. In the experience of the authors, such differences can occur due to the manufacturing

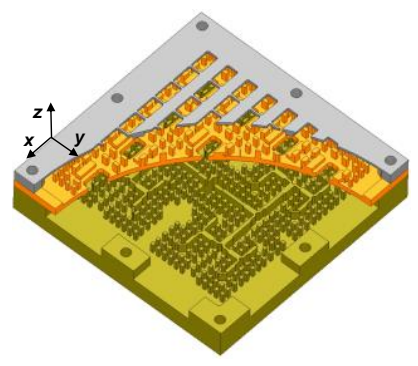

(a)

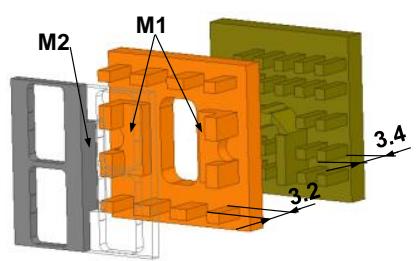

(b)

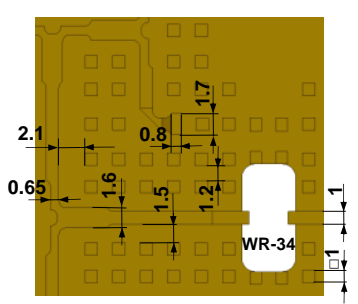

(c)

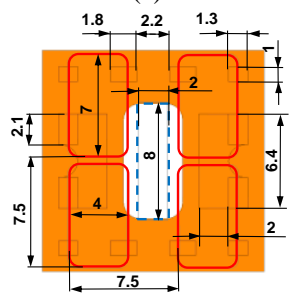

(d)
Fig. 4. Design of the fixed-beam GWG array: (a) assembled structure, (b) $2 \times 2$ subarray, (c) detailed view of the RGW distribution layer with dimensions, (d) enlarged resonator layer. (All dimensions are in millimeters) (a)

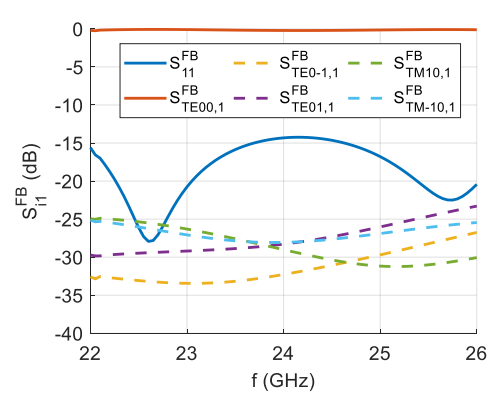

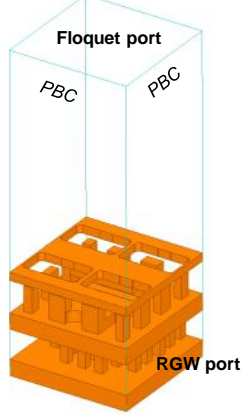

(b)
Fig. 5. Simulated $S$-parameters (a) of the $2 \times 2$ GWG slot subarray (b).

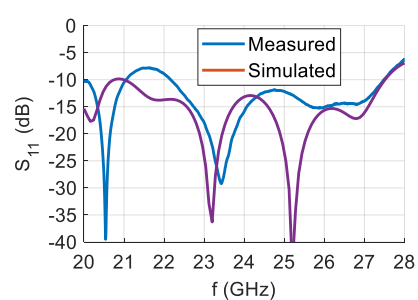

(a)

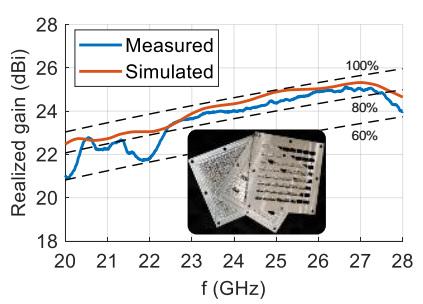

(b)
Fig. 6. GWG array performance: (a) input port reflection coefficient, (b) broadside antenna realized gain, where the dashed lines correspond to the theoretical gain curves for the denoted values of the antenna efficiency.

tolerances that are particularly critical in the area of the RGW layer to the resonator layer transition. Nonetheless, in the frequency band of interest $(23-25 \mathrm{GHz})$, the $S_{11}$ levels are well below the targeted $-10 \mathrm{~dB}$.

The near-field distribution of the $8 \times 8$ GWG array was studied at different distances above the slotted aperture. We found that within the $4-8 \mathrm{~mm}$ distance, the variations of E-field magnitude and phase in the xoy-plane are less than $2.5 \mathrm{~dB}$ and $25^{\circ}$, respectively. Fig. 7 shows the simulated $E_{y}$ field component distribution at $24 \mathrm{GHz}$ and $6 \mathrm{~mm}$ distance above the aperture in the E- and H-plane.

\section{B. Transmitarray Unit Cell}

The TA was developed using multilayer PCB technology. The exploded TA UC structure and the PCB stackup are demonstrated in Fig. 8(a) and 8(b), respectively. As seen, the TA contains 8 layers (7 conducting, 1 empty), with a topology realizing the necessary functionalities as discussed in Section II (Fig. 1). The conducting layers are etched on the core Rogers RO4003C substrates $\left(\varepsilon_{r}=3.55, \tan \left(\delta_{e}\right)=0.0027\right)$, which are bonded using Rogers RO4450B prepregs $\left(\varepsilon_{r}=3.54\right.$,

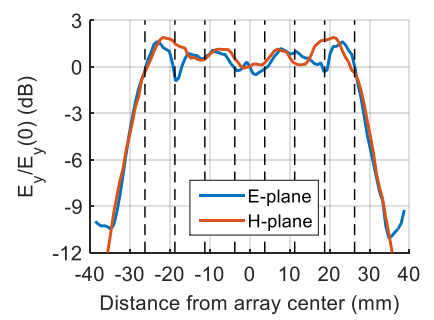

(a)

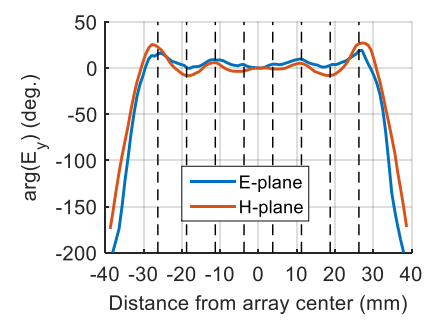

(b)
Fig. 7. Simulated distribution of $E_{y}$ component at $6 \mathrm{~mm}$ above the GWG array aperture, $24 \mathrm{GHz}$ : (a) magnitude, (b) phase. Dashed lines correspond to the center positions of the slots. 
$\left.\tan \left(\delta_{e}\right)=0.004\right)$. The total PCB thickness is $2.5 \mathrm{~mm}$. Detailed views of the main layers are presented in Fig. 9, where layer 1 (Fig. 9(a)) comprises slot elements forming the RX array, which couples to the fixed-beam array. Slots have the H-shape with the inner microstrips. This technique allows having an electrically wide slot $(\sim 0.09 \lambda)$, providing a relatively large bandwidth, while preserving a compact longitudinal size. From the TA side, the slot couples to the inner stripline topology of layer 2 through the rectangular resonator (Fig. 9(b)). Layer 2 also comprises a fixed phase-shifting section realized as a shielded meander stripline. By varying the length $L_{P S}$ we can control the inner phase shift of the UC. The stripline topology of layer 2 is shielded by via group 1 , whose blind viaholes connect layer 1 and layer 4 . The output of the phaseshifting line is connected to the via-hole of via group 3. These through via-holes form a substrate integrated coaxial line (SIC) and guide a high-frequency signal to the topology of layer 8 , which comprises TX radiating elements. In layer 6 , the DC biasing circuitry is connected to the center via of the SIC through the stripline band-stop filter (Fig. 9(c)). Layers 4, 5,7 play the role of the ground planes for the stripline parts and the TX radiating element. Layer 3 is empty, i.e. it does not contain any conducting elements. A detailed view of the TX radiating element is presented in Fig. 9(d). This element is a cavity-backed slotted patch resonator fed in its geometrical center. As opposed to the designs from [34] - [36] the patch has additional $\Gamma$-shaped slot segments surrounding ground viaholes of the SIC. The $\Gamma$-slot configuration affects the patch input impedance and resonance position. The via-holes of via group 2 connect layer 5 and layer 8 , and thus form the cavity

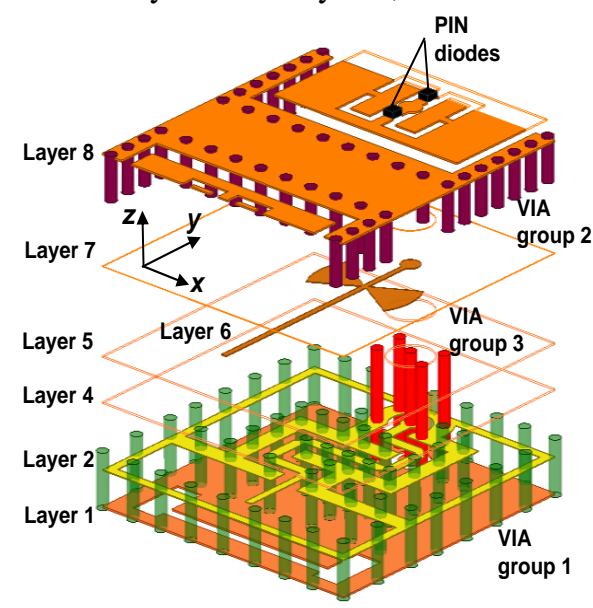

(a)

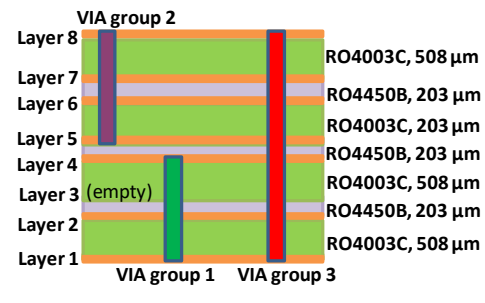

(b)

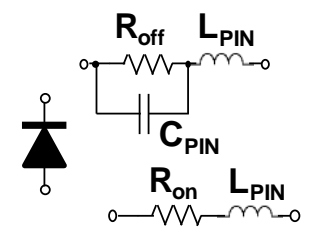

(c)
Fig. 8. TA UC structure. (a) Exploded view; (b) PCB stackup; and (c) PIN diode equivalent models in $\mathrm{ON}$ and OFF states.

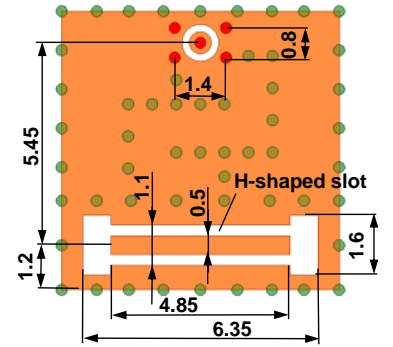

(a)

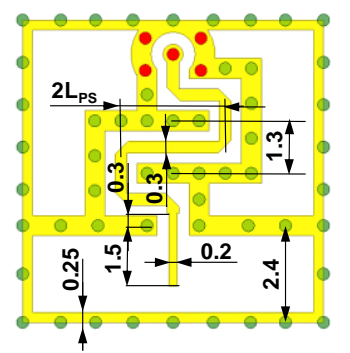

(b)

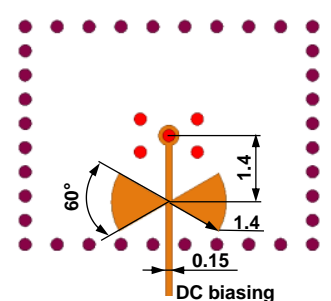

(c)

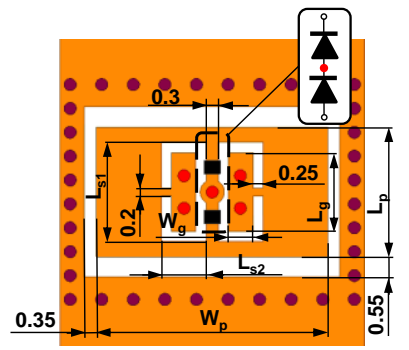

(d)
Fig. 9. Detailed view of the main TA UC layers: (a) layer 1; (b) layer 2; (c) layer 6; (d) layer 8. (All dimensions are in millimeters)

walls for the TX element. Compared to the previous 4-layer designs [34] - [37], the PCB stackup complexity has been increased. The proposed UC employs two groups of blind viaholes, which make it more expensive to manufacture as compared to the reported simple PCB-based structures (e.g., [37]). To a large extent, the increased complexity is the result of the additional functionality that the UC should realize by accommodating the separate shielded layer with the fixed phaseshifting sections. Another two ground layers 5, 7 were added to fully shield the biasing circuitry from the TA radiating parts. This makes the UC performance less sensitive to control lines tracing. On the other hand, results reported in [35], [39] indicate that radiating and biasing parts can be successfully allocated inside the same electromagnetic environment. This provides the direction for further TA structure simplification.

The PIN diodes are integrated inside the $0.2 \mathrm{~mm}$ microstrip gap (Fig.9(d)) of the TX patch. The diodes are oriented in opposite ways with respect to the feeding point and biased simultaneously through the center SIC via. Thus, a dualpolarity control system is needed to realize the 1-bit phase control. In this research, we used AlGaAs PIN diodes MADP000907-14020 from MACOM. The equivalent circuit models of the diode in ON and OFF states are given in Fig. 8(c). The forward $(\mathrm{ON})$ operation regime was set at $10 \mathrm{~mA}$ current, which corresponds to $1.37 \mathrm{~V}$ of forward biasing voltage. To find the lumped elements parameters of the equivalent circuits, we first characterized the diode mounted inside the $0.2 \mathrm{~mm}$ gap of a microstrip line with $0.3 \mathrm{~mm}$ width. In this case, we used an ad hoc microstrip TRL calibration setup. Next, the diode electromagnetic model was adapted for the operation inside the TX element structure. For this purpose, we have employed a well-established waveguide simulator technique [34] - [36], [43]. As the radiation centers of the RX and TX elements are shifted at $5.45 \mathrm{~mm}$ along the $y$-axis, it is impractical to build a simulator for the full UC. Due to this, we stud- 


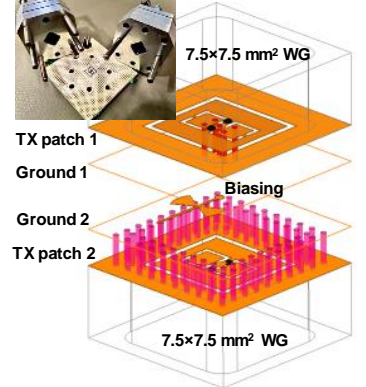

(a)

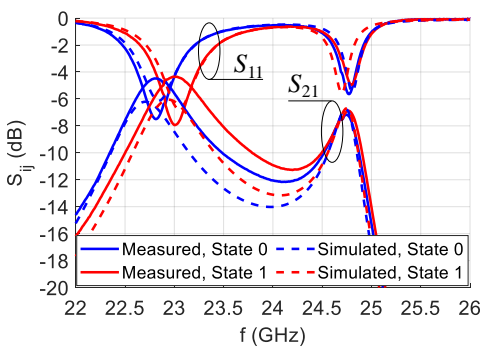

(b)
Fig. 10. Waveguide simulator setup: (a) exploded view of the intermediate UC structure; (b) comparison of measured and simulated results.

ied an intermediate structure comprising two directly connected TX elements and the biasing circuitry, located inside a $7.5 \times 7.5 \mathrm{~mm}^{2}$ square waveguide (Fig. 10(a)). The structure has the following main dimensions: $L_{p}=4.75 \mathrm{~mm}, W_{p}=5 \mathrm{~mm}$, $L_{s 1}=2.05 \mathrm{~mm}, L_{s 2}=1.75 \mathrm{~mm}, L_{g}=1.55 \mathrm{~mm}, W_{g}=1.25 \mathrm{~mm}$. Other dimensions are given in Fig. 9(d). The fabricated simulator prototype is shown in the inset of Fig. 10(a). Fig. 10(b) compares the measured and simulated $S$-parameters to support a generally good agreement of their frequency responses. Eventually, the following diode's parameters were extracted: $R_{\text {on }}=3.3 \Omega, R_{\text {off }}=20 \mathrm{k} \Omega, C_{P I N}=33 \mathrm{fF}, L_{P I N}=0.1 \mathrm{nH}$.

After obtaining the PIN diode equivalent model, the UC structure was optimized through the full-wave analysis in Ansys HFSS. At the first stage, we considered the elements separately by introducing auxiliary stripline ports between the TX and RX elements. The RX port is allocated close to the output of the rectangular resonator, whereas the TX port is introduced nearby the SIC. We have set PBC at the side faces of the UC and Floquet ports at $20 \mathrm{~mm}$ above and beneath the

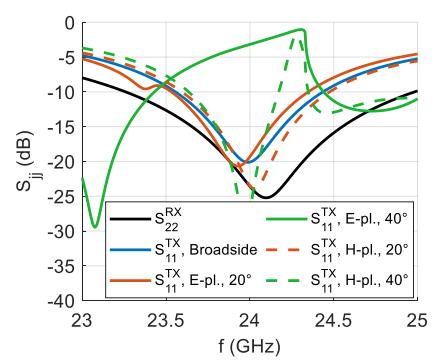

(a)

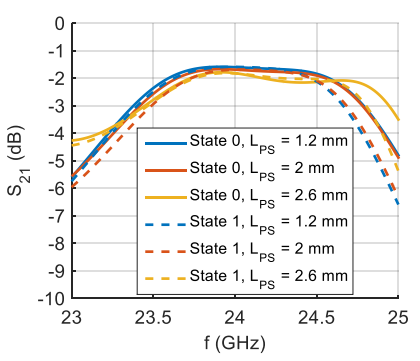

(c)

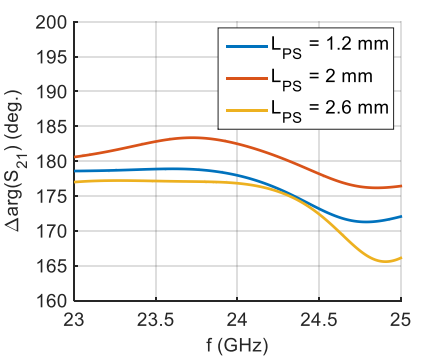

(b)

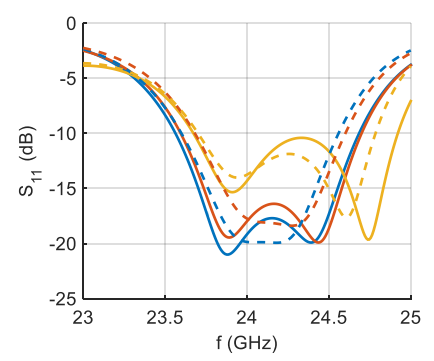

(d)
Fig. 11. Simulated $S$-parameters. (a) Separate performance of the RX (broadside) and TX (various beam steering directions, State 0 ) elements. The full TA UC performance for different values of $L_{P S}$ : (b) phase difference between two UC states; (c) transmission coefficient; (d) reflection coefficient ( $\mathrm{RX}$ side). structure along $z$-axis. The RX element was simulated with the broadside illumination, which corresponds to its operation conditions. The simulated reflection coefficient at the stripline port side is shown in Fig. 11(a). As expected, $S^{R X}{ }_{22}$ demonstrates a relatively wideband frequency response with $24 \mathrm{GHz}$ central frequency.

The TX radiators during beam steering operate in a varying electromagnetic environment, which cannot be represented rigorously by $\mathrm{PBC}$ due to the 1-bit phase-shifting realized by the TX element structure reconfiguration. Nevertheless, according to the previous studies [34] — [40], the Floquet analysis of a reconfigurable UC in both states provides the desired level of assessment of TA beam-steering performance. The TX element structure was optimized with the goal to achieve the best impedance matching for $\theta_{s}=(0-40)^{\circ}$ range. The final design parameters are the following: $L_{p}=2.75 \mathrm{~mm}$, $W_{p}=5.1 \mathrm{~mm}, L_{s 1}=2.45 \mathrm{~mm}, L_{s 2}=1.1 \mathrm{~mm}, L_{g}=1.95 \mathrm{~mm}$, $W_{g}=0.6 \mathrm{~mm}$. The simulated results for different beam steering directions can be seen in Fig. 11(a) for State $0\left(0^{\circ}\right.$ state). At the maximum beam-steering angles in both cardinal planes, the scan blindness is observed near the appearance of the first grating lobe. Of course, the finite reconfigurable TX aperture will not demonstrate such a sharp mismatching, which is the characteristic only of the periodic structure [44]. However, from the given analysis, we expect a radiation performance degradation in the E-plane for $\theta_{s}>30^{\circ}$.

Finally, the full TA UC was simulated with the PBC corresponding to the broadside operation. We considered several values of $L_{P S}$ from the range required to realize the phase distribution (8) with $F=60 \mathrm{~mm}$. The results are presented in Fig. 11(b) - (d) (port 1 denotes the Floquet port at the RX side). The simulated insertion loss at the central frequency is below $1.8 \mathrm{~dB}$, and the $3-\mathrm{dB}$ transmission bandwidth is around $1.65 \mathrm{GHz}$ spreading from 23.2 to $24.85 \mathrm{GHz}$.

\section{NFC of the GWG Array and Transmitarray}

The NFC mechanism was studied using the UC models from Sections III.A, B and the full-wave model in Fig. 12. The latter comprises the coupled configuration of the $2 \times 2 \mathrm{GWG}$ subarray and 4 RX UCs. Each RX element is connected to the auxiliary port (Section III.B). The sidewall boundary conditions are set to the broadside PBC. Since both the fixed-beam and TA RX UCs have non-ideal free-space impedance matching, the optimum value of $D_{\text {coup }}$ can be found considering the full-system reflection coefficient $S^{N F C}{ }_{11}$ (ports numbering is

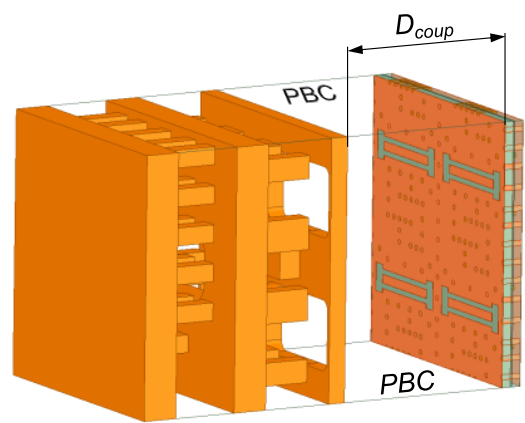

Fig. 12. Full-wave model of the coupled $2 \times 2$ GWG slot subarray and four RX unit cells of the TA. 


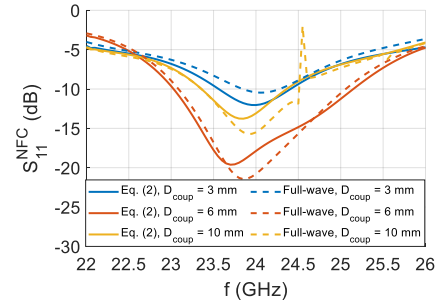

(a)

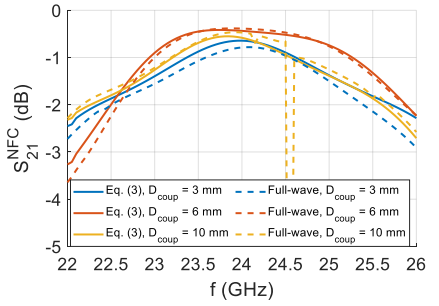

(b)
Fig. 13. $S$-parameters of the near-field coupled configuration computed from (2), (3) and simulated using the full-wave model in Fig. 12.

according to Fig. 2). In Fig. 13, we demonstrate the results from (2), (3) in comparison with the full-wave model performance. Note that for the full-wave case $S^{N F C}{ }_{21}=\left(S^{N F C 1}{ }_{21}+\right.$ $\left.S^{N F C 2}{ }_{21}+S^{N F C 3_{21}}+S^{N F C 4}{ }_{21}\right) / 2$, where transmission coefficients in the parentheses correspond to each of four slot RX elements in Fig. 12. As expected, for smaller values of $D_{\text {coup }}$ there is some results discrepancy due to the interaction of the reactive fields. When $D_{\text {coup }}>5 \mathrm{~mm}$, the results are nearly identical. However, for larger $D_{\text {coup }}$ the higher-order Floquet mode (which is, to a small extent, excited by the $2 \times 2$ GWG subarray) can resonate inside the coupling region. This phenomenon is illustrated by the sharp increase of $S_{11}$ for $D_{\text {coup }}=10 \mathrm{~mm}$. From the given parametric study, we found that $D_{\text {coup }}=6 \mathrm{~mm}$ provides the widest matching bandwidth. This optimal value was used for the PAA system design. It can be noted that the optimal $D_{\text {coup }}$ corresponds to the broadside resonance condition of the equivalent Fabry-Perot resonator [45], which is formed by the coupled arrays. As $D_{\text {coup }} / \lambda<1$, no resonances can be supported in the coupling region for the oblique incidence.

\section{EXPERIMENTAL STUDY OF THE FULL $8 \times 8$ ARRAY PROTOTYPE AND RESULTS DISCUSSION}

The fully assembled antenna system is presented in Fig. 14, where Fig. 14(b) shows a side view of the coupling region. The total thickness of the PAA is $21.2 \mathrm{~mm}(1.7 \lambda)$. The measured $S_{11}$ at WR-34 input is demonstrated in Fig. 15(a) for beam steering in the E- and the H-plane. The measured -10$\mathrm{dB}$ impedance bandwidth spends a $23.5-25.2 \mathrm{GHz}$ range.

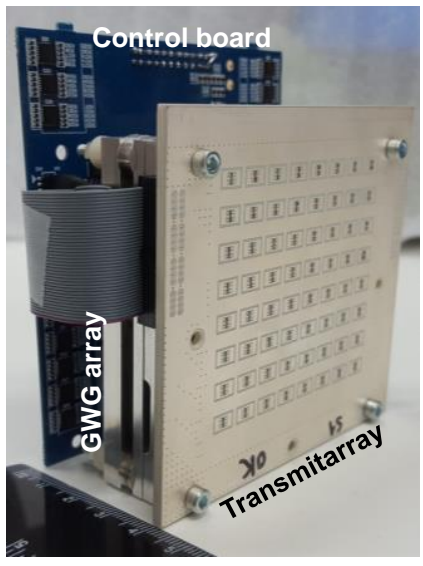

(a)

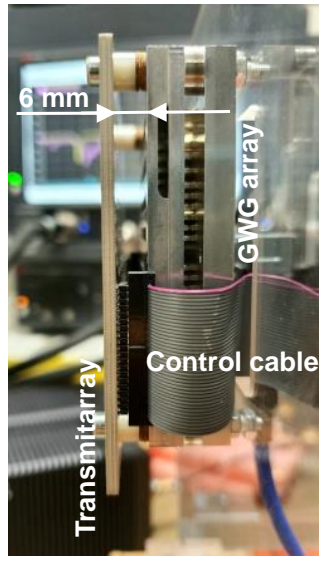

(b)
Fig. 14. Photographs of the $8 \times 8$ PAA system experimental prototype: (a) assembled system; (b) side view of the coupling region.

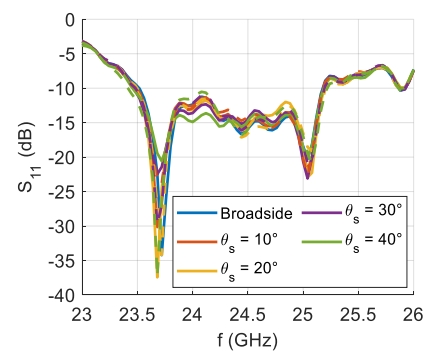

(a)

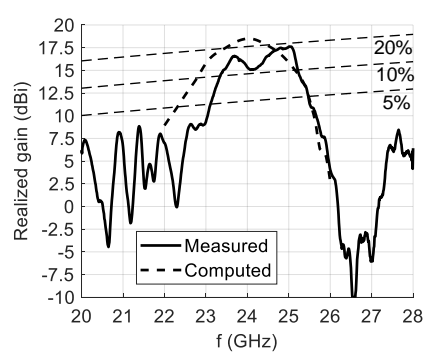

(b)
Fig. 15. Performance of the $8 \times 8$ PAA: (a) measured input reflection coefficient for the beam steering in the E- (solid lines) and the H-plane (dashed lines); (b) measured and computed broadside co-polarized realized gain.

The observed $350 \mathrm{MHz}$ bandwidth shift to the higher frequencies is due to the manufacturing tolerance of the TA via-holes, which has led to the $50 \mu \mathrm{m}$ diameter increase of all holes. As a result, the equivalent inductance of the central SIC via-hole has been reduced, shifting the TX patch resonance to the higher frequencies. This phenomenon has been verified through the full-wave simulations. The data is omitted here due to space limitations. For the considered application case, this shift has been found acceptable. The effect of the beam steering on the $S_{11}$ levels is weak with the worst values of $-10.5 \mathrm{~dB}$ at $\sim 24 \mathrm{GHz}$. Such scan behavior originates due to the equiphase excitation system of the GWG array (the RGW distribution and the resonator layers), which partly blocks the fixedbeam array input from the beam-steering reflections of the TA.

Radiation characteristics of the experimental antenna were studied during far-field measurements inside an anechoic chamber. We found that an acceptable (in terms of gain scan loss and SLL) beam-steering performance can be realized inside $23.7-25.1 \mathrm{GHz}$ bandwidth. Fig. 16 presents the measured steered-beam far-field patterns at several frequencies that are also overlaid with the numerical data as obtained using the TA radiation model. Measurements demonstrate that in the H-plane the beam can be successfully steered up to $\theta_{s}=$ $40^{\circ}$, whereas in the E-plane the SLL increases more significantly when steering towards $\theta_{s}=40^{\circ}$. The useful E-plane beam steering range was determined as $\pm 30^{\circ}$. This effect is expected and has been predicted by the UC Floquet analysis (Section III.B). In the main lobe region, the analytical method agrees fairly well with the measured data, except for some dips in the relative main lobe level observed for the measured results. The main differences are observed for SLLs, where the worst measured SLL is around $-7 \mathrm{~dB}$. In overall, the achieved SLL is similar to the published for the integrated-feed TA [23]. Furthermore, the analytical model predicts that the maximum SLL will decrease as the PAA aperture increases, since the averaging of phase quantization errors over the TX aperture will be more effective. Additional reduction in SLLs can be achieved if we use a fixed-beam array with isolated channels that prevents array elements coupling through a power distribution system.

The measured relative cross-polarization level within the main lobe is below $-23 \mathrm{~dB}$ at all frequencies. 
In Fig. 15(b), the measured realized gain frequency response is compared with the computed data for the broadside beam-steering regime. To compute the PAA gain $(G)$, we approximated the far field by a superposition of active TX element patterns [26], where each TX element was excited through the NFC system (Fig. 2) cascaded with time delay lines:

$$
\begin{gathered}
G=\frac{4 \pi d_{x} d_{y}}{\lambda^{2} N_{x} N_{y}}\left|\sum_{i=1}^{N_{x}} \sum_{j=1}^{N_{y}} S_{T E 00,1}^{i j}\right|^{2} L_{R G W}, \\
S_{T E 00,1}^{i j}=\frac{S_{21}^{N F C} S_{T E 00,1}^{T X i j} \exp \left(-\gamma_{S L} L_{i j}\right)}{1-S_{22}^{N F C} S_{11}^{T X i j} \exp \left(-2 \gamma_{S L} L_{i j}\right)},
\end{gathered}
$$

where $S^{T X i j} T E 00,1$ denotes the TX element transmission coefficient to the $\mathrm{TE}_{00}$ Floquet mode, which determines the copolarized radiation for $\varphi_{s}=0$; the $(i, j)$ TX element $S$-matrix is chosen according to the phase state of the $(i, j) \mathrm{UC} ; \gamma$ is the delay line propagation constant, $L_{i j}$ is the length of the $(i, j)$ delay line. The interaction with the RGW power distribution system is considered by adding the insertion loss multiplier $L_{R G W}=-0.7 \mathrm{~dB}$. The measured gain demonstrates in-band oscillations with the maximum dip of $1.4 \mathrm{~dB}$ around $24 \mathrm{GHz}$. Comparing the gain response in Fig. 6(b) for the fixed-beam GWG array alone with the gain in Fig. 15(b) for the full assembled antenna system, we see that the TA introduces a

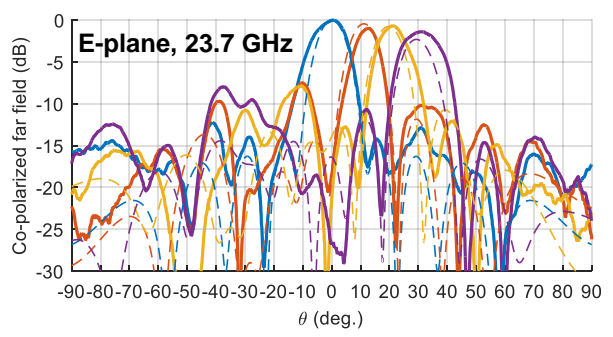

(a)

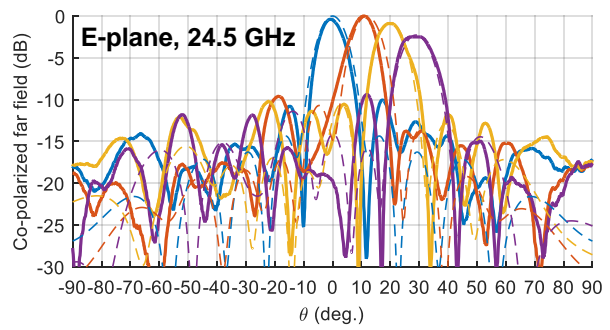

(c)

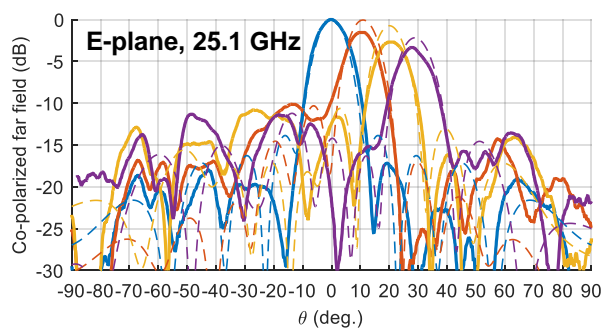

(e)
$6.5-8 \mathrm{~dB}$ loss. This additional loss contribution to the antenna efficiency includes the $1.8 \mathrm{~dB}$ insertion loss of the TA $\mathrm{UC}$, the $3.5-4.5 \mathrm{~dB}$ directivity drop due to the 1-bit phase shifting [35], [42], and extra efficiency degradation due to non-desired interaction effects between two arrays. The latter effects can be explained as follows: (i) the reflected power of the TX aperture is bounced back by the aperture of the GWG array that is not perfectly impedance matched (especially at off-broadside illumination angles); (ii) as the result, the redirected power passes through the fixed phase shifting sections several times (due to multiple interactions) and again subjected to the 1-bit phase quantization leading to the degradation of the TX aperture phase distribution. These multiple-interaction effects are the main reason of the oscillations (Fig. 15(b)) and are similar to those observed in beam-steering phased-array fed reflector systems [46], where additional complication in our case occurs due to the 1-bit phase quantization. In-depth analysis of these effects has been left for future studies, while the key observation for this work is that both arrays should be initially optimized for the minimum reflection loss in the desired frequency bandwidth and beam-steering range.

In Table I, we summarized the main performance metrics of the aforementioned PAA architectures employing the TA technology. As can be seen, the 1-bit approach provides the lowest UC insertion loss. At the same time, the main drawback of the TAs with the 1-bit phase quantization is the notable directivity loss of at least $4 \mathrm{~dB}$. For the focal source architecture, aperture efficiency further degrades due to the illumi-

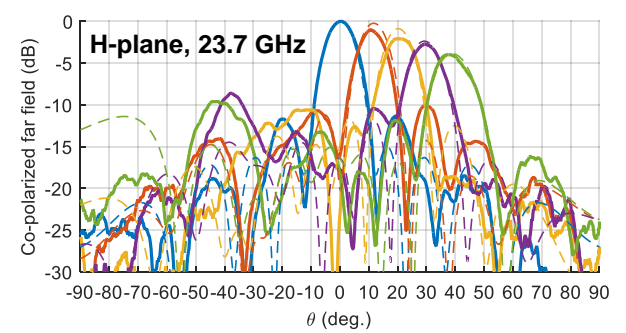

(b)

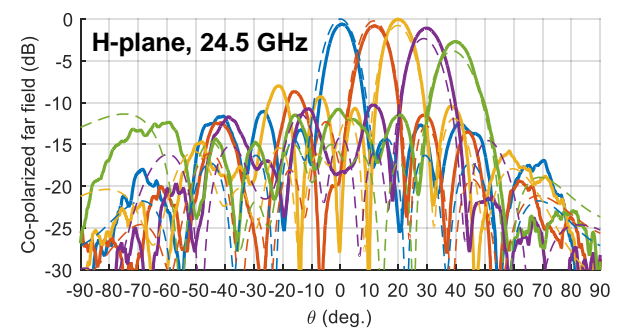

$\theta$ (deg.

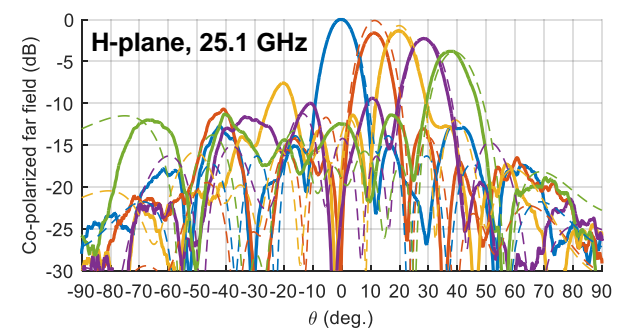

(f)

Fig. 16. Measured (solid lines) and computed (dashed lines) co-polarized far-field patterns of the $8 \times 8$ PAA at different frequencies for $(0-30)^{\circ}$ and $(0-40)^{\circ}$ beams steering sectors $\left(10^{\circ}\right.$ step) in the E- and $\mathrm{H}$-plane, respectively. 
nation taper and spill-over loss. In contrast, the NFC architecture is free from both these issues and thus, at mm-wave frequencies, provides a very attractive combination of high total antenna efficiency, low-profile structure, and high power handling capability. Another advantage is that it allows the whole structure to be readily scaled up in terms of the number of array antenna elements. This can be done by either redesigning the fixed-beam array power distribution network (along with the associated modification of the TA inner phase shift distribution), or by simply using identical $N \times N$ sub-arrays with an appropriate TX elements control method. This technique is expected to realize the far-field performance similar to TAs with multiple focal sources [38], [39].

The main direction of the NFC architecture improvement is to increase its bandwidth. The achieved bandwidth of different TA architectures is compared in Table 1 . The -10-dB impedance bandwidth of the TAs with a focal source is defined based on the UC frequency responses. Presently, the NFC architecture is more narrowband as compared to conventional TAs with a focal source; this is mainly attributed to the TX elements impedance mismatch and mutual interaction effects of two arrays. Addressing the former issue would require the enhancement of the TX radiating element. The latter can be done by improving the patch topology so as to reduce the shunting effect of the SIC ground pins. Alternatively, PIN diodes with a lower capacitance value can also enhance the bandwidth. Furthermore, the TX elements E-plane decoupling would result in the beam-steering improvement that is critical at higher frequencies. The cavity-backed patch resonators decoupling can be effectively achieved by employing the planar EBG structures [47].

\section{CONCLUSION}

The proposed PAA system architecture based on the TA NFC to the fixed-beam array has been examined. It employs the plane-wave near field of the fixed-beam array for the excitation of the reconfigurable TA and can be designed through the following key steps. Step 1: Development of the fixedbeam array and its radiative near field analysis. Step 2: Synthesis of the TA phase distribution and the UC optimization. Step 3: Integration of the full coupled array system, where the optimum $D_{\text {coup }}$ can be found by the minimization of the reflection loss in the desired operation bandwidth.

The above-described design flow has been successfully verified through an example of the $8 \times 8 \mathrm{~mm}$-wave beamsteering antenna system, where the high-efficiency fixed-beam GWG array was coupled to the PIN diode-loaded reconfigurable printed TA with the 1-bit phase control. Our experimental study shows the $23.5-25.2 \mathrm{GHz}-10-\mathrm{dB}$ impedance bandwidth, the $23.3-25.3 \mathrm{GHz} 3-\mathrm{dB}$ gain bandwidth and achievable beam-steering range of $\pm 40^{\circ}$ in the $\mathrm{H}$-plane and $\pm 30^{\circ}$ in the E-plane. To the authors best knowledge, this is the first reported prototype realizing the PAA architecture with the NFC to the reconfigurable TA.

Generalization/application of the presently implemented NFC approach is possible to other fixed-beam PAA types, such as printed patch arrays, waveguide arrays, leaky-wave slot arrays, etc.

\section{ACKNOWLEDGMENT}

Authors would like to thank Professor Rob Maaskant (Chalmers University of Technology) and Professor Sergey Skobelev (Moscow Institute of Physics and Technology) for the fruitful discussions regarding the TA model development. We also wish to express our gratitude to Mr. Eduard Mozharov (Bauman Moscow State Technical University) for his help in anechoic chamber measurements.

\section{References}

[1] "On mm-wave technologies for NR," Ericsson LM, Stockholm, Sweden, R4-1642262016. [Online]. Available: http://www.3gpp.org/ftp/TSG_RAN/WG4_Radio/TSGR4_79/Docs/R4164226.zip

[2] I. Kadri, A. Petosa, and L. Roy, "Ka-band Fresnel lens antenna fed with an active linear microstrip patch array," IEEE Trans. Antennas Propag., vol. 53, no. 12, pp. 4175-4178, Dec. 2005.

[3] M. K. Saleem, H. Vetticaladi, M. A. S. Alkanhal, and M. Himdi, "Lens antenna for wide angle beam scanning at $79 \mathrm{GHz}$ for automotive short range radar applications," IEEE Trans. Antennas Propag., vol. 65, no. 4, pp. 2041-2046, Apr. 2017.

[4] F. K. Schwering, "Millimeter wave antennas," Proc. IEEE, vo1.80, no.1, pp. 92-102, Jan. 1992.

[5] R. J. Mailloux, Phased array antenna handbook, 3rd ed., Norwood, MA: Artech House, 2018.

[6] S. V. Hum and J. Perruisseau-Carrier, "Reconfigurable reflectarrays and array lenses for dynamic antenna beam control: a review," IEEE Trans. Antennas Propag., vol. 62, no. 1, pp. 183-198, Jan. 2014.

[7] R. Maaskant, W. A. Shah, A. U. Zaman, M. Ivashina, and P.-S. Kildal, "Spatial power combining and splitting in gap waveguide technology," IEEE Antennas Wireless Propag. Lett, vol. 26, no. 7, pp. 472-474, June 2016.

[8] Y. Li, K.-M. Luk, "60-GHz substrate integrated waveguide fed cavitybacked aperture-coupled microstrip patch antenna array," IEEE Trans. Antennas Propag., vol. 63, no. 3, pp. 1075-1085, March 2015

[9] J. F. Xu, Z. N. Chen, X. M. Qing, and W. Hong, "Bandwidth enhancement for a $60 \mathrm{GHz}$ substrate integrated waveguide fed cavity array antenna on LTCC," IEEE Trans. Antennas Propag., vol. 59, no. 3, pp. 826-832, Mar.2011.

[10] R. Carrillo-Ramirez and R. W. Jackson, "A highly integrated millimeterwave active antenna array using $\mathrm{BCB}$ and silicon substrate," IEEE Trans. Microw. Theory Tech., vol. 52, no. 6, pp. 1648-1653, June 2004.

[11] A. U. Zaman and P. S. Kildal, "Wide-band slot antenna arrays with single-layer corporate-feed network in ridge gap waveguide technology," IEEE Trans. Antennas Propag., vol. 62, no. 6, pp. 29923001, June 2014.

[12] D. Zarifi, A. Farahbakhsh, A. U. Zaman, and P. S. Kildal, "Design and fabrication of a high-gain $60 \mathrm{GHz}$ corrugated slot antenna array with ridge gap waveguide distribution layer," IEEE Trans. Antennas Propag., vol. 64, no. 7, pp. 2905-2913, July 2016.

[13] D. Zarifi, A. Farahbakhsh, and A. U. Zaman, "A gap waveguide-fed wideband patch antenna array for $60-\mathrm{GHz}$ applications," IEEE Trans. Antennas Propag., vol. 65, no. 9, pp. 4875-4879, July 2017.

[14] M. S. Sorkherizi, A. Dadgarpour, and A. A. Kishk, "Planar highefficiency antenna array using new printed ridge gap waveguide technology," IEEE Trans. Antennas Propag., vol. 65, no. 7, pp. 3772-3776, Jul. 2017.

[15] J. Zhang, X. Zhang, and A. A. Kishk, "Broadband $60 \mathrm{GHz}$ antennas fed by substrate integrated gap waveguides," IEEE Trans. Antennas Propag., vol. 66, no. 7, pp. 3261-3270, Jul. 2018.

[16] A. Farahbakhsh, D. Zarifi, A. U. Zaman, " 60-GHz groove gap waveguide based wideband $\mathrm{H}$-plane power dividers and transitions: For use in high-gain slot array antenna ", IEEE Trans. Microw. Theory Tech.V. 65, no. 11, pp. 4111-4121, Nov. 2017.

[17] E. Baghernia, A.-R. Sebak, and M. R Chaharmir, "Groove gap waveguide slot array antenna in millimeter wave band", In Proc. of Antenna Technology and Applied Electromagnetics (ANTEM) 2018 18th International Symposium on, Waterloo, ON, Canada, 2018, pp. 1-2. 
[18] M. Al Sharkawy and A. A. Kishk, "Wideband Beam-Scanning Circularly Polarized Inclined Slots Using Ridge Gap Waveguide," in IEEE Antennas and Wireless Propagation Letters, vol. 13, pp. 1187-1190, 2014.

[19] M. Vukomanovic, J. L. Vazquez-Roy, O. Quevedo-Teruel, E. RajoIglesias, and Z. Sipus, "Gap waveguide leaky-wave antenna," IEEE Trans. Antennas Propag., vol. 64, no. 5, pp. 2055-2060, May 2016.

[20] L. Wang, J.-L. Gomez-Tornero, E. Rajo-Iglesias, and O. QuevedoTeruel, "Low-dispersive leaky-wave antenna integrated in groove gap waveguide technology," IEEE Trans. Antennas Propag., vol. 66, no. 11, pp. 5727-5736, Nov. 2018.

[21] C. Bencivenni, T. Emanuelsson, and M. Gustafsson, "Gapwaves platform integrates 5G mmWave arrays," Microwave Journal, vol. 62, no. 2, Feb. 2019.

[22] M. V. Ivashina, T. Eriksson, R. Rehammar, Shi Lei, C. Bencivenni, and R. Maaskant, "A 1-D steerable beam slotted waveguide antenna employing non-conventional aperiodic array architecture for mm-wave line-ofsight MIMO," In Proc. of 2019 IEEE International Conference on Microwaves, Communications, Antennas and Electronic Systems (COMCAS 2019), Tel Aviv, Israel, 2019, paper accepted.

[23] J. G. Nicholls and S. V. Hum, "Full-space electronic beam-steering transmitarray with integrated leaky-wave feed," IEEE Trans. Antennas Propag., vol. 64, no. 8, pp. 3410-3422, Aug. 2016.

[24] C.-T. Cheung, R. Tsai, R. Kagiwada, and D. B. Rutledge, "V-band transmission and reflection grid amplifier packaged in waveguide," in IEEE MTT-S Int. Dig., 2003, vol. 3, pp. 1863-1866.

[25] T. Lou, X.-X. Yang, H. Qiu, Z. Yin, and Steven Gao, "Compact dualpolarized continuous transverse stub array with 2-D beam scanning," IEEE Trans. Antennas Propag., vol. 67, no. 5, pp. 3000-3010, May 2019.

[26] A. K. Bhattacharyya, Phased Array Antennas: Floquet Analysis, Synthesis, BFNs and active array systems. Hoboken, NJ: John Wiley \& Sons, Inc., 2006.

[27] W. Y. Yu, W. Li, A. Elsherbeni, and Y. Rahmat-Samii, Advanced Computational Electromagnetic Methods and Applications. Norwood, MA: Artech House, 2015.

[28] C. K. Balanis, Antenna Theory: Analysis and Design. 3rd ed., Hoboken, NJ: John Wiley \& Sons, Inc., 2005.

[29] C.-C. Cheng and A. Abbaspour-Tamijani, "Study of 2-bit antennafilter-antenna elements for reconfigurable millimeter-wave lens arrays," IEEE Trans. Microw. Theory Tech., vol. 54, no. 12, pp. 4498-4506, Dec. 2006

[30] C.-C. Cheng, B. Lakshminarayanan, and A. Abbaspour-Tamijani, "A programmable lens-array antenna with monolithically integrated MEMS switches," IEEE Trans. Microwaves Theory Tech., vol. 57, no. 8, pp. 1874-1884, Aug. 2009.

[31] L. Boccia, I. Russo, G. Amendola, and G. D. Massa, "Multilayer antenna-filter antenna for beam-steering transmit-array applications," IEEE Trans. Microw. Theory Tech., vol. 60, no. 7, pp. 2287-2300, Jul. 2012.

[32] J. Y. Lau and S. V. Hum, "Reconfigurable transmitarray design approaches for beamforming applications," IEEE Trans. Antennas Propag., vol. 60, no. 12, pp. 5679-5689, Dec. 2012.

[33] J. Y. Lau and S. V. Hum, "A planar reconfigurable aperture with lens and reflectarray modes of operation," IEEE Trans. Antennas Propag., vol. 58 , no. 12 , pp. $3547-3555$, Dec. 2010

[34] A. Clemente, L. Dussopt, R. Sauleau, P. Potier, and P. Pouliguen, "1-bit reconfigurable unit cell based on pin diodes for transmit-array applications in X-band," IEEE Trans. Antennas Propag., vol. 60, no. 5, pp. 2260-2269, May 2012.

[35] A. Clemente, L. Dussopt, R. Sauleau, P. Potier, and P. Pouliguen, "Wideband 400-Element electronically reconfigurable transmitarray in $\mathrm{X}$ band," IEEE Trans. Antennas Propag., vol. 61, no. 10, pp. 5017-5027, Oct. 2014.

[36] L. Di Palma, A. Clemente, L. Dussopt, R. Sauleau, P. Potier, and P. Pouliguen, "1-bit reconfigurable unit-cell for Ka-band transmitarrays," IEEE Antennas Wireless Propag. Lett, vol. 15, pp. 560-563, 2016.

[37] B. D. Nguyen and C. Pichot, "Unit-cell loaded with PIN diodes for 1-bit linearly polarized reconfigurable transmitarrays," IEEE Antennas Wireless Propag. Lett, vol. 18, no. 1, pp. 98-102, Jan. 2019.

[38] L. Di Palma, A. Clemente, L. Dussopt, R. Sauleau, P. Potier, and P. Pouliguen, "Reconfigurable transmit-array antenna with multiple focal sources," In Proc. of The 8th European Conference on Antennas and
Propagation (EuCAP 2014), The Hague, Netherlands, 2014, pp. 23082312.

[39] L. Di Palma, A. Clemente, L. Dussopt, R. Sauleau, P. Potier, and P. Pouliguen, "Circularly-polarized reconfigurable transmitarray in Kaband with beam scanning and polarization switching capabilities," IEEE Trans. Antennas Propag., vol. 65, no. 2, pp. 529-540, Feb. 2017.

[40] L. Di Palma, A. Clemente, L. Dussopt, R. Sauleau, P. Potier, and P. Pouliguen, "Experimental characterization of a circularly-polarized 1bit unit-cell for beam steerable transmitarrays at Ka-Band," IEEE Trans. Antennas Propag., vol. 67, no. 2, pp. 1300-1305, Feb. 2019.

[41] R. J. Mailloux, "Array grating lobes due to periodic phase, amplitude and time delay quantization," IEEE Trans. Antennas Propag., vol. 32, pp. 1384-1368, Dec. 1984.

[42] A. Vilenskiy, M. Makurin, and C. Lee, "Phase distribution optimization for 1-bit transmitarrays with near-field coupling feeding technique", In Proc. of The 14th European Conference on Antennas and Propagation (EuCAP 2020), Copenhagen, Denmark, 2020, unpublished (accepted).

[43] P. W. Hannan and M. A. Balfour, "Simulation of a phased-array antenna in waveguide," IEEE Trans. Antennas Propag., vol. 13, no. 3, pp. 342353, May 1965.

[44] M. H. Awida, A. H. Kamel, and A. E. Fathy, "Analysis and design of wide-scan angle wide-band phased arrays of substrate-integrated cavitybacked patches," IEEE Trans. Antennas Propag., vol. 61, no. 6, pp. 3034-3041, Jun. 2013.

[45] A. P. Feresidis and J. C. Vardaxoglou, "High-gain planar antenna using optimized partially reflective surfaces," Proc. Inst. Elect. Eng., Microw. Antennas Propag., vol. 148, no. 6, pp. 345-350, Feb. 2001.

[46] O. Iupikov, R. Maaskant, and M. Ivashina, "Fast and accurate analysis of reflector antennas with phased array feeds including multiple reflections between feed and reflector," IEEE Trans. Antennas Propag., vol. 62, no.7, pp. 3450-3462, July 2014

[47] A. R. Vilenskiy, V.I. Litun, and K. V. Lyulyukin, "Wideband beam steering antenna array of printed cavity-backed elements with integrated EBG structure," IEEE Antennas Wireless Propag. Lett, vol. 18, no. 2, pp. 245-249, 2019.

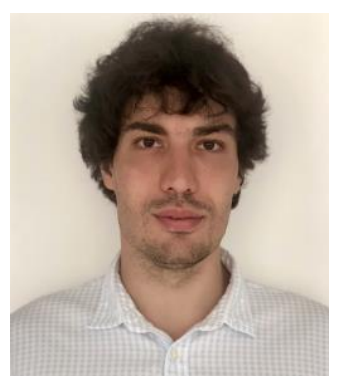

Artem R. Vilenskiy (M'11) received the specialist degree in radioengineering and the Ph.D. degree in antennas and microwave devices from Bauman Moscow State Technical University, Moscow, Russia, in 2011 and 2014, respectively.

In 2011, he joined Electromagnetics Solutions Lab of Samsung Research Institute Russia, Moscow, Russia, where he currently holds an expert engineer position in RF Sensor Part. From 2015 to 2019 he was an Associate Professor at the Radio-Electronic Systems and Devices Department of Bauman Moscow State Technical University. Since 2019 he has been a Researcher with Antenna Systems Group, Chalmers University of Technology, Gothenburg, Sweden. His research interests include computational electromagnetics, periodic structures, antenna arrays, microwave devices and wireless power transmission systems.

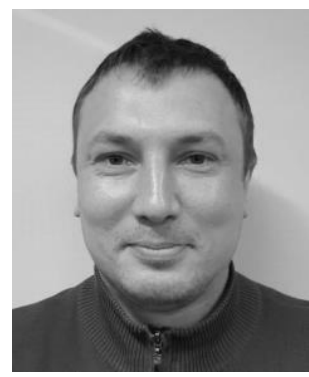

Mikhail N. Makurin received the B.S., M.S. in applied physics and Ph.D. degree in radiophysics from the Moscow Institute of Physics and Technology, Moscow, Russia, in 2002, 2004, and 2010, respectively. In 2010, he joined to the Samsung Research Institute Russia, Moscow, Russia. He is now with the RF sensor 
part of Algorithm Research Team, Samsung Research Institute Russia.

His research interests include antennas, analytical electromagnetics, microwave circuits, controllable materials for microwave and wireless power transmission systems.

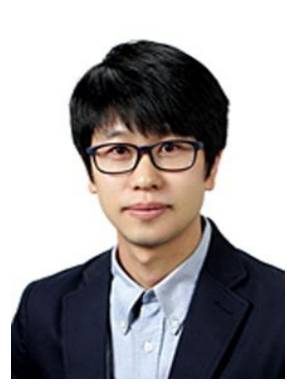

Chongmin Lee received the Ph.D. degree in Electrical engineering from the Soongsil University of Seoul, Korea, in 2013.

In 2013, he joined Samsung Electronics, Suwon, Korea. He is now a Staff Engineer in Samsung Research of Samsung Electronics, Seoul, Korea. He has been involved in research on various topics, such as resonators and transceiver systems for wireless power transfer, $\mathrm{RF} / \mathrm{mm}$-wave communication systems, metamaterial-based resonator structures for voltage-controlled oscillators.

His current research is focused on array antenna systems and control algorithms for long-distance wireless power transfer.

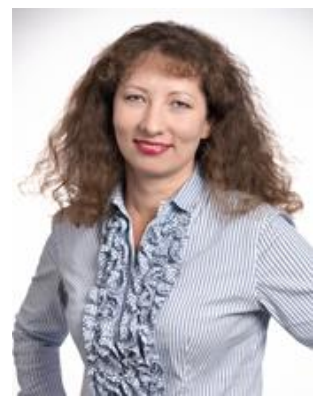

Marianna V. Ivashina (M'11-SM'13) received a Ph.D. in Electrical Engineering from the Sevastopol National Technical University (SNTU), Ukraine, in 2001. From 2001 to 2010 she was with The Netherlands Institute for Radio Astronomy (ASTRON), where she carried out research on innovative phased array technologies for future radio telescopes, such as the Square Kilometer Array. In 2002-2003, she also stayed as a Visiting Scientist with the European Space Agency, ESTEC, where she studied multiple-beam array feeds for the satellite telecommunication system Large Deployable Antenna.

In January 2011, she joined Chalmers University of Technology (Gothenburg, Sweden), where is currently Full Professor and the Head of the Antenna Systems Group at the Department of Electrical Engineering. Her current research interests include antenna array systems, integration of antennas with active electronic components, synthesis of aperiodic arrays, and other unconventional array architectures, reflector antennas, and focal plane array feeds. She has published extensively on the above topics, having authored/co-authored over 130 journal and conference papers. She has received several scientific awards [including the URSI Young Scientists Award for GA URSI, Toronto, Canada (1999), APS/IEEE Travel Grant, Davos, Switzerland (2000), the 'Best team contribution' Paper Award at the ESA Antenna Workshop (2008), the EU FP7 Marie Curie Actions International Qualification Fellowship (2009), Best Paper Award at the IEEE COMCAS Conf., Tel-Aviv, Israel (2019)], and numerous research project funding grants from Swedish and European funding agencies.

Prof. Ivashina is presently an Associate Editor of the IEEE Transactions on Antennas and Propagation and a Board member of the European School of Antennas (ESoA). 